\title{
Envie de soigner et commerce de dignités
}

\author{
D. Gross
}

Cela fait trente-cinq ans que je suis médecin. Je suis sur le point de me corriger de ce vilain défaut. Seule l'imminence de la phase terminale de ma carrière me donne le culot et la vanité de vous faire part de l'opinion d'un généraliste de banlieue, attentif à l'évolution de la médecine, des malades et des médecins que j'observe si possible avec «le jugement assez droit avec l'esprit le plus simple».

La médecine a beaucoup progressé. Les maladies, celles du moins qui figurent dans les traités, sont abordées avec de meilleures armes: mieux connues, plus vite identifiées, plus efficacement traitées grâce surtout au progrès des techniques et des médicaments. Les spécialistes sont mieux formés et plus nombreux donc plus accessibles. Ce constat n'est pas partagé par tous les patients si j'en crois la floraison des médecines parallèles. Je n'y vois pas seulement un désaveu mais une recherche naïve de la magie et de l'irrationnel dont la médecine s'éloigne toujours plus et qui semble si nécessaire à beaucoup de patients dans l'art de guérir. J'aurais voulu utiliser ces médecines enjolivées comme appoint mais je dois admettre que leur efficacité ne dépasse guère l'effet placebo.

Les malades ont changé aussi: plus curieux, plus exigeants, pas vraiment mieux informés. Il y a toujours ceux qu'on appelait les «chroniques» en levant les yeux au ciel, dont on ne sait plus très bien ce qu'ils font dans la consultation, et qui nous reposent ou nous irritent selon les disponibilités de l'agenda. J'ai vu surtout arriver en masse des patients qui ont décidé un jour de quitter les rangs ternes des bien-portants pour se précipiter joyeux dans le gai carrousel du «disease biz». Ces gens, gestionnaires avisés de l'économie familiale, ne pouvaient admettre de payer chaque mois de lourdes sommes sans en retirer le moindre bénéfice. Ils ont apprivoisé la maladie et les médecins et sont facilement devenus des patients réguliers au titre d'une saine conception de la prévention des maladies. Et la Nature, dans son infinie sagesse, semble avoir créé le cholestérol pour leur fournir un éternel sujet de conversation. Ce phénomène n'est pas nouveau bien sûr mais ce qui était une caricature désopilante du temps de Knock était en fait une géniale prémonition de la situation actuelle. Bien qu'un peu surpris de cet engouement, je m'en suis accommodé, sous l'influence de mon comptable et des psychiatres pour qui le seul fait de consulter dénote une intéressante psychopathologie. En moi, le soignant répugne à ces gesticulations mais le petit commerçant est satisfait de cet afflux. Et pour les patients, la solidarité, belle valeur à la base du principe de l'assurance, a une limite quantitative au-delà de laquelle le citoyen généreux se sent pousser des ailes de pigeon.

Les généralistes ont changé. L'entendue de leur domaine s'est rétrécie à mesure que les spécialistes devenaient plus compétents et disponibles, tandis que leur rôle de conseiller ou de confident s'est renforcé. Un peu frustrés de ne gagner que leur vie alors que certains spécialistes faisaient fortune, ils ont cru à l'efficacité de leurs mandataires et se sont laissé imposer une interminable nomenclature de leurs gestes appelée TARMED, censée donner une image plus claire de leurs prestations et distribuer leurs revenus plus équitablement. Pauvres confrères!, élèves appliqués par nature, qui se sont astreints à des cours d'initiation et ont noirci des pages de notes sous la direction des Tarmedologues FMH, ont comparé leurs comptabilités avec angoisse et se sont précipités à des cours de formation pour augmenter leurs «dignités» et ainsi étoffer leurs factures de quelques points supplémentaires. Les premiers résultats de cet amphigouri pernicieux ne semblent pas apporter un début de solution aux vrais problèmes.

Les politiciens, affolées et incapables de cerner les données, paraissent voir dans le pouvoir des médecins la source de tous les maux. Ils ont commencé par supprimer l'obligation des langues anciennes, dans l'idée que la pléthore de médecins ainsi induite les rendrait dociles. Ils ont cautionné le TARMED, imaginant amener ces rapaces à résipiscence. Puis ils ont interdit l'ouverture de nouveaux cabinets médicaux. Enfin, ils souhaitent donner aux caisses-maladie le pouvoir de choisir les médecins. Autant donner aux loups le pouvoir de choisir les bergers! Ces décisions incohérentes ont abouti à transformer l'orgueilleux humaniste en boutiquier fébrile. Est-ce un progrès?

Si je puis me permettre d'aventurer une esquisse de tendance, je jugerais utile de redonner aux malades le goût d'être bien-portants et aux médecins l'envie de soigner les gens dans une ambiance économique plus sereine. Ambitieux programme, dont les solutions ... mais il y a des bornes à l'effronterie d'un de vos 28000 administrés, par ailleurs abstentionniste honteux mais résolu ...

Dans quelques mois, j'aurai sans doute beaucoup de peine à quitter mes malades mais, grâce au TARMED, je cesserai sans regrets de faire commerce de mes dignités. 